Voix et Images

volxetimages

\title{
De Hiroshima à Éroshima
}

Une érotique de la bombe atomique en forme de haiiku selon Dany Laferrière

From Hiroshima to Éroshima

The Erotics of the Atom Bomb as Haiku According to Dany Laferrière

\section{Cécile Hanania}

Volume 31, numéro 1 (91), automne 2005

Figures et contre-figures de l'orientalisme

URI : https://id.erudit.org/iderudit/011926ar

DOI : https://doi.org/10.7202/011926ar

Aller au sommaire du numéro

Éditeur(s)

Université du Québec à Montréal

ISSN

0318-9201 (imprimé)

1705-933X (numérique)

Découvrir la revue

Citer cet article

Hanania, C. (2005). De Hiroshima à Éroshima : une érotique de la bombe atomique en forme de haïku selon Dany Laferrière. Voix et Images, 31(1), 75-87. https://doi.org/10.7202/011926ar

\section{Résumé de l'article}

L’Orient n'est pas au coeur des préoccupations de Dany Laferrière, dont les ouvrages s'inscrivent pour la plupart dans une géographie retraçant un parcours personnel, de son île natale, Haïti, à son premier lieu d'exil, Montréal. Pourtant, son second texte, Éroshima, est tissé de motifs issus de la culture japonaise. L'auteure analyse ici le statut ambigu de cette thématique asiatique qui véhicule un grand nombre de stéréotypes. Elle s'attache à montrer comment le narrateur livre une réflexion sur la prégnance du cliché et tente de la déjouer en adoptant un mode poétique étranger à la pensée occidentale. 


\section{DE HIROSHIMA Ã ÉROSHIMA.}

Une érotique de la bombe atomique en forme de haïku selon Dany Laferrière

$$
++
$$

CÉCILE HANANIA

Western Washington University

\section{RÉSUMÉ}

L'Orient n'est pas au cœur des préoccupations de Dany Laferrière, dont les ouvrages s'inscrivent pour la plupart dans une géographie retraçant un parcours personnel, de son île natale, Haïti, à son premier lieu d'exil, Montréal. Pourtant, son second texte, Éroshima, est tissé de motifs issus de la culture japonaise. L'auteure analyse ici le statut ambigu de cette thématique asiatique qui véhicule un grand nombre de stéréotypes. Elle s'attache à montrer comment le narrateur livre une réflexion sur la prégnance du cliché et tente de la déjouer en adoptant un mode poétique étranger à la pensée occidentale. 
Le choix d'une étude sur Éroshima ${ }^{1}$ pourrait paraître à première vue insolite dans un dossier consacré à la représentation de l'«Orient» au Québec. La géographie cosmopolite du texte, qui évoque des lieux occidentaux (de New York à Berlin en passant par Rome), et la "québécité» d'emprunt de son auteur d'origine haïtienne, Dany Laferrière, ne posent pas cet ouvrage, a priori, en peinture exemplaire de contrées orientales. D'autant que le récit, pour entraîner le lecteur dans un tour du monde échevelé et chaotique, met en scène un narrateur indolent et sédentaire qui se refuse à quitter son lit. Si Charles Baudelaire chantait jadis une «splendeur orientale» faite de «divans profonds», la position de dilection, horizontale, du protagoniste, enfoncé dans son futon, ne manifeste pas une «invitation au voyage ${ }^{2}$ ». Quoi qu'il en soit de ce paradoxe initial et du lieu d'origine de son auteur, le texte a pour point d'ancrage l'avenue du Parc, au cœur de Montréal, et pour fil conducteur l'Asie, et tout particulièrement le Japon, à travers deux références antithétiques que reflète le titre valise mêlant éros à Hiroshima: l'initiation amoureuse du narrateur par de jeunes Japonaises et le bombardement de la ville nippone, le 6 août 1945.

Eroshima est la seconde œuvre de Dany Laferrière. Sa composition et son contenu, singuliers par bien des aspects dans la production de l'auteur ${ }^{3}$, doivent toutefois beaucoup à l'ouvrage précédent: Comment faire l'amour avec un Nègre sans se fatiguer ${ }^{4}$. Par sa teneur érotique, Éroshima constitue un diptyque, très certainement motivé par des raisons commerciales, avec ce retentissant succès paru deux ans auparavant.

Bien que sous-titré «roman", Éroshima n'a ni l'unité, ni la cohérence d'une narration romanesque. Constitué de seize parties ou tableaux disparates, de longueurs très inégales (une à quatre-vingts pages), le texte mélange, dans une confusion qui frise parfois le coq-à-l'âne, réflexions historiques, saynètes érotiques et entretiens fictifs. Au dire de Laferrière ${ }^{5}$, Éroshima est le récit que son narrateur entreprenait d'écrire dans Comment faire l'amour sous le titre: «Paradis du dragueur nègre». Récit qu'il aurait initialement inséré dans les dialogues et que son éditeur aurait ensuite supprimé.

Moins qu'une suite ou un complément à Comment faire l'amour, Éroshima apparaît davantage comme un volet antithétique. Après la sexualité volcanique et débridée d'un immigré haïtien, il expose l'érotisme subtil et méthodique de Japonaises raffinées et met en scène un narrateur plus effacé, confronté à des

$$
+++
$$

1 Dany Laferrière, Éroshima, Montréal, VLB éditeur, 1987. Désormais, les références à ce roman seront indiquées par le sigle $E R$, suivi du folio, et placées entre parenthèses dans le texte. 2 Charles Baudelaire, Les fleurs du mal, Paris, Éditions Gallimard, coll. «Poésie», 1990 [1857]. 3 Contrairement à tous les autres récits de Laferrière, Éroshima ne renvoie à aucun élément autobiographique immédiatement identifiable. En outre, il est le seul ouvrage où l'évocation de l'«Asie», même fantasmatique, tienne une place centrale. Ce n'est qu'avec Je suis fatigué (Montréal, Lanctôt éditeur, 2001 [2000]) qu'un personnage féminin japonais, Kero, déjà mentionné (ÉR, 79 et suivantes), resurgit dans la narration, p. 15-27. 4 Dany Laferrière, Comment faire l'amour avec un Nègre sans se fatiguer, Montréal, VLB éditeur, 1985, abrégé désormais en Comment faire l'amour. 5 Id., J'écris comme je vis (entretiens avec Bernard Magnier), Genouilleux, Éditions La Passe du vent, 2000, p. 152. 
partenaires dominatrices et violentes, comme pour se dédouaner du caractère un tantinet machiste et hâbleur de l'ouvrage précédent. Il rompt également avec un certain manichéisme ethnique. Le Montréal WASP auquel se heurtait l'immigré antillais dans Comment faire l'amour laisse place à une ville plus métissée où les couleurs se troublent, et où, par la grâce de l'onomastique amoureuse nippone, l'amant «nègre» anonyme est baptisé Tosei, soit «pèche verte» (ÉR, 32).

Cet ouvrage, par ses évocations cosmopolites, par la situation de son auteur et la posture singulière de son narrateur, reprend et subvertit les divisions entre Orient et Occident, dominant et dominé, ailleurs et ici, etc. D'un point de vue géographique (selon une division eurocentrique), nous n'avons pas affaire au regard d'un Occidental sur l'Orient, ni à celui d'un Oriental sur l'Occident. Dans une perspective narrative, il ne s'agit ni d'un récit de voyage, ni d'un récit d'exil. Didactiquement, le texte ne prétend ni à l'érudition, ni à l'information, ni même à une quelconque connaissance. «Je ne sais rien du Japon, et le Japon ne sait rien de moi», telle est la déclaration du narrateur à la fin du récit ( $E R, 171)$. Boutade coutumière à Laferrière ou aphorisme de penseur à rapprocher d'une connaissance socratique contenue dans l'affirmation: « Je ne sais qu'une chose, c'est que je ne sais rien $^{6} »$ ? Quel que soit le statut de cette ignorance absolue, revendiquée in fine, c'est autour de ce non-savoir que Laferrière joue dans un texte qui, au-delà des clivages culturels qu'il met en scène, est une réflexion sur la valeur et la possibilité de toute représentation.

Singulier sur la scène littéraire montréalaise et marginal dans l'œuvre de Laferrière, Éroshima n'en compose pas moins avec les représentations séculaires et stéréotypées d'une certaine tradition littéraire. Nous verrons laquelle, avant de nous attarder sur le traitement que subissent les références éclectiques au Japon. En dernier lieu, nous analyserons le statut et la fonction du «cliché» dans le texte.

\section{LE JAPONISME}

De l'idéalisation de la vie rurale à la célébration du caractère urbain de Montréal, l'espace québécois et les questions d'identité nationale ont longtemps dominé chez les écrivains du Québec. Laurent Mailhot note qu'un quart des fictions publiées annuellement ont pour cadre Montréal. Les autres se situent dans un axe québécois Nord/Sud, le long du Saint-Laurent, sur le vaste territoire canadien, dans «les steppes abitibiennes, nord-ontariennes» ou dans «le bureaucratique Ottawa ${ }^{7}$ ». Certaines ont pour cadre les États-Unis. Rares sont celles qui sortent des frontières nord-américaines, du moins jusqu'à une période récente. Les années 1980 et 1990 ont vu en effet les horizons littéraires s'ouvrir et se diversifier, grâce à l'apport de ce que l'on a nommé, à partir de La Québécoite ${ }^{8}$ de Régine Robin, la «littérature

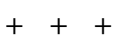

6 Platon, Apologie de Socrate, Paris, Hatier, 1965, p. 21. 7 Laurent Mailhot, La littérature québécoise depuis ses origines, Montréal, TYPO, 2003, p. 229. 8 Régine Robin, La Québécoite, Montréal, Québec/Amérique, 1983. 
migrante ${ }^{9}$ ». De nombreux auteurs expatriés ont substitué les paysages de leur terre natale aux étendues du terroir québécois. L'Asie est ainsi apparue, et notamment le Japon, avec des écrivains tels que Ook Chung et Aki Shimazaki ${ }^{10}$, mais également chez des écrivains nés au Québec. Claude Blouin (déjà versé dans l'Extrême-Orient) publie sa Petite géométrie du cœur ${ }^{11}$ en 1994, et Robert Lepage met en scène des tribulations américano-japonaises, dans une pièce avant-gardiste: The Seven Streams of the River Ota (Les sept branches de la rivière Ota) ${ }^{12}$.

En 1987, toutefois, au moment où paraît le texte de Laferrière, l'«Empire du Soleil-Levant» est une terre quasi inconnue sur la scène littéraire québécoise. Ses évocations sont en grande partie occultées, et rares sont celles qui ont rencontré quelque succès, à l'exception des nouvelles et poèmes de Claude Blouin ${ }^{13}$ et du roman épique de Paul Ohl: Katana ${ }^{14}$. L'étrangeté du Japon pour le lecteur moyen québécois semble telle, que tous les termes nippons employés dans Éroshima sont systématiquement traduits en français ou assortis de paraphrases explicatives. Ainsi, le mot et le mets sushi, aujourd'hui entré dans les dictionnaires et dans les assiettes, doit-il être explicité: "(sorte de riz au vinaigre roulé avec des languettes d'omelette, du cresson, des champignons, des copeaux de courge, et quelques fines tranches de poisson $\mathrm{cru}) »(\bar{E} R, 38)$.

En prenant pour motif récurrent de son récit des éléments de la culture et de l'histoire japonaises, Laferrière se démarque de la production littéraire québécoise (qui ne fait d'ailleurs pas partie de son héritage culturel), mais il n'écrit pas ex nihilo. De même que dans son précédent ouvrage il rompait avec une littérature prude, en renouant par son texte de facture érotique et scandaleuse avec les écrits provocateurs d'auteurs nord-américains tels que Charles Bukowski, Henry Miller, ou l'exilé russe Edouard Limonov, il participe, ou du moins succède, avec Éroshima, à une tradition qui prend racine dans l'Europe du dix-neuvième siècle et son engouement pour l'Orient.

Edward Saïd a amplement décrit l'orientalisme (scientifique et romantique), mais il a abordé essentiellement sa représentation du Proche et du Moyen-Orient ${ }^{15}$. C'est-à-dire, politiquement parlant, d'un Orient dominé, colonisé (en grande partie par les Français), puis perdu (durant les campagnes napoléoniennes) et devenu objet d'une vision nostalgique. De l'Extrême-Orient, il ne dit rien. Le Japon a pourtant occupé une place centrale dans la mode orientaliste entre la fin du dix-neuvième siècle et le début du vingtième. Quand l'isolationnisme traditionnel du pays a été

9 Voir Robert Berrouët-Oriol, «L'effet d'exil», Vice versa, n 17, 1986-1987, p. 20-21. 10 Ook Chung est né au Japon de parents coréens. Voir en particulier, Nouvelles orientales et désorientées, Montréal, l'Hexagone, coll. "Fictions», 1994; id., Contes Buto, Montréal, Boréal, 2003. Aki Shimazaki, Tsubaki, Montréal/Arles, LeméacActes Sud, 1999; id., Hamaguri, Montréal/Arles, Leméac-Actes Sud, 2000; id., Tsubame, Montréal/Arles, Leméac-Actes Sud, 2001; id., Wasurenagusa, Montréal/Arles, Leméac-Actes Sud, 2002. 11 Claude Blouin, Petite géométrie du cœur, Montréal, Boréal, 1994. 12 Robert Lepage, The Seven Streams of the River Ota, Londres, Random House, 1996. 13 Claude Blouin, Du Japon et d'ici, Joliette, Pleins Bords, 1975; id., Dire l'éphémère, Montréal, Boréal, 1983; id., Taire l'essentiel, Montréal, Boréal, 1987. 14 Paul Ohl, Katana. Le roman du Japon, Montréal, Québec/Amérique, 1986. 15 Edward Saïd, Orientalism, New York, Pantheon Books, 1978. 
ébranlé par le traité de Kanagawa, signé en 1854 avec l'amiral Perry, les ports japonais se sont ouverts au commerce international. Les nombreuses expositions universelles américaines et européennes de la fin du dix-neuvième siècle achèvent alors de consacrer cette ouverture, en exposant un grand nombre d'objets d'art japonais et en attirant de nombreux visiteurs nippons.

L'attrait pour les motifs et le style japonais, le "japonisme ${ }^{16}$ ", comme l'a nommé le critique d'art français Philippe Burty, en 1872, a exercé une influence considérable sur l'art occidental. Très prégnant dans la peinture post-réaliste et impressionniste (de James Whistler à Henri de Toulouse-Lautrec, en passant par Auguste Renoir, Vincent Van Gogh, et Mary Cassatt) ainsi que dans l'Art nouveau, il laisse aussi sa marque sur la littérature de l'époque. En témoignent les récits de Pierre Loti, Madame Chrysanthème et Japoneries d'automne ${ }^{17}$ notamment, ou certains essais des frères Edmond et Jules de Goncourt (grands collectionneurs, en outre, de japonaiseries) sur les peintres Kitawaga Utamaro (1891) et Katsushika Hokusaï (1896) en particulier ${ }^{18}$. Mais ce phénomène n'est pas limité à l'Europe. Un même engouement touche les États-Unis. Un des principaux legs de cette mode est le célèbre Madame Butterfly ${ }^{19}$, qui a fait florès.

C'est à cette époque que se forgent certaines représentations stéréotypées du Japon, qu'elles soient esthétiques (épuration des formes, minimalisme, élégance des femmes aux soieries délicates) ou plus psychologiques (esprit samouraï et fascination de la mort, sensualité des geishas, etc.). Cette fascination pour le Japon se poursuit au vingtième siècle, notamment avec les écrits de Paul Claudel ${ }^{20}$. La « vogue» maoïste lui préfère la Chine dans les années 1960, mais le Japon resurgit sporadiquement dans les lettres françaises, chez des intellectuels tels que Roland Barthes avec L'empire des signes $^{21}$ ou Bernard-Henri Lévy et ses Impressions d'Asie ${ }^{22}$.

\section{L'ANTI-VOYAGE}

Laferrière, féru de littérature occidentale, ne peut ignorer les représentations qui l'ont précédé, et, en premier lieu, la manifestation générique de cette fascination: le récit de voyage. L'Orient, quel qu'il soit géographiquement, est un ailleurs plus ou moins lointain que les écrivains ont en général parcouru afin de le décrire. Laferrière

$$
++
$$

16 «Japonisme» est le titre donné à une série d'articles publiés de mai 1872 à février 1873 dans la revue parisienne La Renaissance littéraire et artistique. 17 Pierre Loti, Madame Chrysanthème, Paris, Calmann-Lévy, 1887; id., Japoneries d'automne, Paris, Calmann-Lévy, 1889. 18 Edmond de Goncourt, Outamaro, Hokousaï. L'art japonais au XVIII siècle, Paris, Union générale d'éditions, 1986 [1891 et 1896]. 19 John Luther Long, Madame Butterfly, New York, Century Co., 1898. Il publia également Miss Cherry Blossom of Tôkyô, Philadelphia, J. B. Lippincott Company, 1895. Signalons enfin un précédent tombé aux oubliettes, premier roman, en anglais, d'une écrivaine originaire de Montréal, Winnifred Eaton Babcock, sous le pseudonyme d'Onoto Watanna, The Japanese Nightingale, New York, London, Harper \& Bros., 1901. 20 Voir en particulier, Paul Claudel, "Nô", Euvres en prose, Paris, Éditions Gallimard, coll. «Bibliothèque de la Pléiade», 1989, p. 1167-1176. 21 Roland Barthes, L'empire des signes, Genève, Skira, coll. «Les sentiers de la création», 1970. Désormais, les références à cet essai seront indiquées par le sigle $E S$, suivi du folio, et placées entre parenthèses dans le texte. 22 BernardHenri Lévy, Impressions d'Asie, Paris, Le Chêne/Grasset, 1985. 
joue à dessein de ce nomadisme littéraire dont il prend le contre-pied. Bien que le narrateur d'Éroshima proclame, dans une interrogation goguenarde: "Pourquoi les Asiatiques m'intéressent-elles autant? Parce que c'est loin, l'Asie " (ÉR, 115), cet audelà attrayant ne se visite pas. Le narrateur ne quitte pratiquement jamais l'avenue du Parc où se situe l'«action». De valise, dans le texte, il n'y a que le titre. Dès l'incipit: "Quoi qu'il arrive, je ne bougerai pas du lit», le ton est donné par un être qui affirme, paradoxalement, un immobilisme ${ }^{23}$. Le récit se présente donc comme un anti-voyage. Pourtant, il y a bien «déplacement». Après une première partie statique, constituée essentiellement d'un huis clos amoureux dans un loft, le récit se désunit et se disperse en parties disparates aux titres et aux lieux cosmopolites: Manhattan, Harlem, San Francisco, Berlin, Rome, San Juan, etc., comme autant d'étapes sur un parcours planétaire. Mais ces étapes sont trompeuses. Les villes sont le plus souvent évoquées par des scènes d'intérieur. Il y a bien des fenêtres, récurrentes, mais, contrairement au miroir d'Alice, elles ne se traversent pas. On ne voit jamais, à travers elles, de paysages étrangers. En second lieu, le narrateur intradiégétique du début du récit disparaît totalement de certaines de ces scènes, si bien que l'on ne comprend pas de quel ordre est ce changement spatial, qui a davantage les caractéristiques d'un transport imaginaire relevant d'une ubiquité démiurgique, onirique et narrative que d'un voyage réel. Enfin, ces villes n'ont rien à voir avec un emplacement géographique: "[Elles] n'ont ni visage ni contours propres et ressemblent à des non lieux hybrides ${ }^{24}$.» Elles correspondent à une rencontre ou une évocation d'ordre artistique ou littéraire: Norman Mailer à Manhattan (dans une partie intitulée «Manhattan Kosher» dont le titre, par jeu de mots, renvoie au roman Manhattan Transfer ${ }^{25}$ de John Dos Passos), Jean-Michel Basquiat à Harlem, Alberto Moravia à Rome, ou encore V. S. Naipaul à San Juan ${ }^{26}$. Il n'est pas jusqu'au simple parcours de 128 secondes, en métro montréalais, qui ne se fasse sous les auspices du récit érotique d'André Pieyre de Mandiargues, La motocyclette ${ }^{27}$. Certaines parties sont même constituées uniquement d'une citation. "Pékin sans fin» est extrait d'un entretien de Louis-Ferdinand Céline avec Madeleine Chapsal ${ }^{28}$. Sous le titre «Tombe, Bombe!» s'ouvre une partie composée d'un morceau de conversation entre le journaliste Bayon et le célèbre écrivain américain, Hubert Selby 29.

Le transport relève de la ficelle littéraire, les écrivains font office de villes et la citation tient lieu de voyage et de description. Comme si parler de lieux étrangers était toujours précédé d'une fiction, d'une représentation écran, d'une fenêtre symbolique. Si des passages littéraires se substituent aux passages géographiques qu'en est-il alors de la référence au Japon?

$$
++
$$

23 Ce non-désir renvoie certainement à la pratique zen: méditation sans objet en posture rigide héritée de la position de Bouddha. Tout mouvement, et a fortiori tout voyage, serait ainsi une sorte de méconnaissance de la spiritualité zen: contemplation de l'espace du dedans. 24 Ursula Mathis-Moser, Dany Laferrière, la dérive américaine, Montréal, VLB éditeur, 2003. 25 John Dos Passos, Manhattan Transfer, New York/Londres, Harper \& Brothers, 1925. 26 Ce passage est repris tel quel dans Le goût des jeunes filles, Montréal, VLB éditeur, 1992. 27 André Pieyre de Mandiargues, La motocyclette, Paris, Éditions Gallimard, 1963. 28 Madeleine Chapsal, Envoyez la petite musique, Paris, LGF, coll. «Biblio Essais», 1990 [1984]. 29 Bayon, Selby de Brooklyn (entretien avec Hubert Selby), Paris, Christian Bourgois, 1983. 


\section{LA « JAPONITÉ »SELON LAFERRIÈRE}

Le Japon n'apparaît jamais comme un espace réel, un territoire défini et parcouru (si ce n'est à la fin de l'ouvrage en tant que lieu vague où le narrateur s'imagine perdu), mais comme une entité matérialisée par des objets hétéroclites et incarnée dans des corps féminins. Le tout, importé et transplanté à Montréal, obéit à deux principes: l'antagonisme et le lieu commun. Le narrateur le dit clairement: «je ne m'intéresse qu'aux clichés» (ÉR, 163).

Ces éléments japonais s'affichent de façon ostentatoire, dès le paratexte, avec une reproduction, en première de couverture, de l'estampe du peintre Katsukawa Shunsho (1726-1792): «Dame Musaraki». Ils se poursuivent par d'abondantes allusions au fil du texte à divers éléments de la «culture» japonaise : futon, kimono, bambou, cérémonie du thé et bains mixtes, et par la présence abondante d'un lexique exotique (du grec exotikos: "étranger») consacré au thé (ÉR, 81) et à la nourriture: tofu, sushi, fugu, soja, saké et autres concombres au gingembre. Ces références éclectiques ne sont pas le Japon, mais ses signes les plus manifestes. Elles témoignent d'une "japonité», telle que la percevait naguère Roland Barthes: moins qu'une «essence orientale» (ES, 7), le sémiologue y voyait l'antithèse d'une rusticité gauloise $^{30}$.

Laferrière, Haïtien du Québec, n'oppose pas comme Barthes jadis, l'épaisseur campagnarde du potage français $(E S, 21)$ à la «légèreté du bouillon» translucide nippon $(E S, 22)$, la lourdeur de la friture $(E S, 34)$ à la tempura dentelée de farine (ES, 36), le plantureux de la nourriture occidentale au "minuscule» du "comestible» japonais $(E S, 24)$, mais la «vulgarité nord-américaine» $(E R, 14)$ au «raffinement oriental» $(\bar{E} R, 14)$, la «transe vaudou » à la "sérénité Zen» (ÉR, 16), la "végétation haïtienne luxuriante» au «jardin sec épuré», «la sexualité volcanique des brousses » à «la sensualité minutieuse de Kyôto » (ER, 17). En un mot, le macho mal dégrossi des Caraïbes, à la gracile nippone qui est pour lui «l'élégance suprême» (ÉR, 164).

Chez les deux auteurs, l'autre n'apparaît pas simplement différent mais contraire. Le Japon n'incarne pas l'ailleurs mais l'antipode, au prix d'un binarisme difficile à dépasser, où n'est projetée sur lui que l'image inversée de soi-même. Ainsi, à travers ces multiples composantes japonaises, Laferrière semble perpétuer une dichotomie séculaire entre lourdeur occidentale et subtilité orientale. Mais cette division sectaire et stéréotypée n'a pas simplement une valeur parodique, comme dans Comment faire l'amour où les pires clichés étaient mis en scène et satirisés. Le texte est beaucoup plus ici qu'un jeu sur les clivages ethniques ou culturels.

En dehors de ces éléments éclectiques, le motif asiatique est conditionné par un mythe plus scabreux : le fantasme sexuel lié à une supposée sensualité japonaise assortie d'une technique hors-pair. L'Orient, dans l'imaginaire occidental, a de tout temps évoqué la lascivité, la luxure et la volupté. Aujourd'hui encore, combien d'ouvrages alléchants, associant le plaisir des sens à un savoir-faire tout oriental,

$+++$ 
s'affichent aux devantures des librairies, à vocation pas forcément érotique: du Jardin parfumé ${ }^{31}$, manuel d'érotologie arabe, au Dictionnaire des postures amoureuses ${ }^{32}$ en passant par L'érotisme indien ${ }^{33}$ et L'art taoiste du massage sensuel ${ }^{34}$. Le Japon, à travers l'ambiguité de ses geishas (peintes en poupées peu farouches livrées à la convoitise lubrique de tous ${ }^{35}$ ) et la promiscuité de ses bains mixtes qui heurtèrent la pudeur des premiers voyageurs, n'échappe pas à cette représentation. La vision d'une «Asie galante» semble d'ailleurs fortement inscrite dans l'imaginaire haïtien, à en juger par les textes d'un René Depestre, fervent adepte d'un "géolibertinage ${ }^{36}$ » qui met à contribution des jeunes femmes chinoises et japonaises ${ }^{37}$.

Dans le texte de Laferrière, à défaut de géographie, le lecteur est convié à une chorégraphie amoureuse. Mais cette chorégraphie relève davantage du combat guerrier que de la symbiose pacifique. Au prix d'un subterfuge culturel (qui en dit long sur la charge fantasmatique du texte), elle est associée au célèbre traité indien: le Kâma-Sûtra ${ }^{38}$. Ce guide amoureux, en dehors de son contenu licencieux, est une typologie pratique de l'amour qui classe et décompose les postures et les figures. Laferrière fait de même. Cependant, chaque fois que le Kâma-Sûtra est cité, c'est pour évoquer la contiguïté du plaisir et de la douleur. Le texte insiste ainsi sur «la pression des ongles » qui est « de huit sortes» $(\bar{E} R, 51)$, ou sur les différents types de «morsures» $(\bar{E} R, 52)$. Cette coupure du plaisir est symbolisée par la parcellisation d'une première partie qui, loin de livrer au lecteur de façon linéaire et prodigue les ébats amoureux des protagonistes, les dissèque, tout au contraire, en cent cinquante fragments et quelques coups de ciseaux tranchants, qui viennent ça et là ensanglanter les chairs.

L'ambiguïté de cette sexualité faite de «milles douleurs exquises» $(\bar{E} R, 80)$ est reprise dans l'ambivalence de la référence au Japon. Les scènes érotiques alternent avec des allusions aux ravages de la bombe atomique et associent intimement dans cet Orient, l'éros à l'horreur.

Ce procédé qui consiste à entrecouper les ébats amoureux de renvois à Hiroshima n'est pas sans rappeler le célèbre scénario de Marguerite Duras, et l'adaptation cinématographique d'Alain Resnais: Hiroshima mon amour (1960) ${ }^{39}$, auxquels le titre et le texte de Laferrière doivent sans doute beaucoup ${ }^{40}$. À l'instar du scénario durassien, ouvert sur un couple enlacé dans un lit, dans la ville de Hiroshima, le narrateur d'Éroshima déclare avoir eu l'idée de son texte en imaginant «un jeune couple en train de faire l'amour dans la ville d'Hiroshima, le matin de

$$
+++
$$

31 Muhammad Ibn Umar Al-Nafzâwî, Le jardin parfumé, manuel d'érotologie arabe du cheikh Nefzaoui, Paris, Philippe Picquier, coll. «Poche», 2002. 32 Jacques Cotin, Dictionnaire des postures amoureuses, Paris, Philippe Picquier, coll. «Poche», 2001. 33 Alka Pande et Lance Dane, L'érotisme indien, Paris, La Maisnie-Tredaniel, 2002. 34 Stephen Russel et Jurgen Kolb, L'art taoïste du massage sensuel, Paris, Courrier du livre, 1993. 35 Pierre Loti, Japoneries d'automne, op. cit., p. 63. 36 René Depestre, Alléluia pour une femme jardin, Paris, Éditions Gallimard, Coll. «Folio», 1981 [1973], p. 121. 37 On consultera en particulier id., "Un rêve japonais", Éros dans un train chinois, Paris, Éditions Gallimard, 1990. Titre qui semble faire écho au «éros dans le train» (ÉR, 116). 38 Shri Vâtsyâyana, Kâma-sûtra, Paris, LGF, coll. «Poche», 2002. 39 Marguerite Duras, Hiroshima mon amour, Paris, Éditions Gallimard, 1960. 40 Même si le narrateur se défend d'avoir vu le film tiré de cet ouvrage dans Comment faire l'amour, op. cit., p. 115, la prétérition fait office de confession. 
l'explosion atomique, en 1945. Et la Bombe tombe au moment même où ils parviennent à l'orgasme» (ÉR, 167).

Cette coïncidence entre explosion orgasmique et atomique est le leitmotiv du récit. Dès la dédicace à l'actrice américaine Rita Hayworth, qui donna son nom à la première bombe $\mathrm{H}$ envoyée au-dessus de l'atoll de Bikini (en 1946), le rapprochement entre le sexe et la mort est signifié ${ }^{41}$. Il se retrouve sémantiquement dans tout le texte, à travers la polysémie et la récurrence du mot «bombe» qui qualifie aussi bien une belle femme qu'une arme destructrice, et dans le mélange constant des champs lexicaux de l'érotisme et de la guerre. Si bien que l'on ne sait plus, dans ce lit où le rôle du narrateur revient à «faire l'amour avec un samouraï dans un corps de geisha» (ERR, 80), si les corps s'ébattent ou se battent, s'ils sont complémentaires ou incompatibles. Le futon est un lieu agonistique et la fusion, comme dans le nucléaire, est vue en terme d'explosion. Le motif japonais et l'éros cruel qui en découle ${ }^{42}$, où pulsions masochistes et sadiques, telles qu'elles sont mises en scène par Laferrière («Moi sado, elle maso» [ÉR, 80]), ont l'air de deux vocables nippons, semblent servir d'archétype et de prétexte à une séparation irréductible entre soi et l'autre ${ }^{43}$.

\section{UNE ESTHÉTIQUE DU CLICHÉ}

Il est significatif, à cet égard, que le seul «lieu» japonais évoqué de façon récurrente dans le récit soit Hiroshima. Ailleurs paradoxal, puisque absent et jadis détruit, il apparaît comme une référence par excellence anti-exotique. Si l'Orient est un topos, Hiroshima serait plutôt un a-topos, un non-lieu, un lieu que l'on ne saurait voir et dont on ne peut parler. "Tu n'as rien vu à Hiroshima», tel est le célèbre leitmotiv du scénario durassien, affirmant l'impossibilité de témoigner d'un événement si tragique. Il en va de même ici. Non seulement de Hiroshima, on ne voit rien, mais le seul écrit explicitement mentionné sur la ville naguère détruite, est le témoignage d'un hibakusha (survivant de la catastrophe), aveugle et muet. Le grand-père de l'une des maîtresses du narrateur a composé un poème motivé par un oxymore qui parle «d'une lumière qui rend aveugle. Lumière noire» $(E R$, 49). «Sombre clarté» qui semble prendre le contre-pied d'une création terrestre annoncée dans la Genèse par un jaillissement lumineux: "Et la lumière fut», et une séparation du jour et des ténèbres.

$$
++
$$

41 Parallèle obsessionnel que l'on trouve déjà de façon récurrente dans Comment faire l'amour, op. cit., et plus tard dans Cette grenade dans la main du jeune Nègre est-elle une arme ou un fruit?, Montréal, VLB éditeur, 1993. 42 Et qui n'est sans doute pas étranger à une iconographie teintée de fantasmes, mêlant fascination de la mort kamikaze, hara-kiri des samouraïs, ou encore réalisation cinématographique érotico-sadique, tel que le très célèbre Empire des sens de Nagisa Oshima (1976). 43 Cette séparation relève toutefois d'une interprétation occidentale. Elle reprend l'antagonisme psychique inhérent à l'humain tel que le décrit Sigmund Freud («Au-delà du principe de plaisir", Essais de psychanalyse, Paris, Payot, 1981 [1920], p. 41-115) dans le combat entre ce qu'il nomme Eros et Thanatos: l'instinct de vie et l'instinct de mort. 
Aussi n'est-il guère étonnant que la seule partie explicitement consacrée à Hiroshima, porte un titre anti-génésique: «L'apocalypse n'est qu'un mauvais moment à passer.» (ÉR, 157). Elle est constituée d'une citation du photographe japonais Hiromi Tsuchida, qui a consacré vingt ans de sa vie à saisir sur pellicule les stigmates de la guerre et du désastre atomique:

Quand j'essaie de me demander ce qui m'a amené à Hiroshima, je crois que c'est à
la face cachée, sombre, de mon esprit que je le dois, à mon égoïsme d'auteur.
Pourrais-je même communier avec les souffrances des victimes, qu'en serait-il du
résultat? Tout ce que je perçois, c'est le profond fossé qui existe entre les victimes
de cette Bombe atomique et les gens ordinaires. Il me faut reconnaître que cette
collection de photos ne suffit pas à combler ce fossé. Me reste-t-il encore quelque
chose à faire à ce propos, sinon admettre la honte de mon point de vue d'artiste.
$(\bar{E} R, 159-160)$

Le photographe, qui n'a pour témoigner des conséquences du bombardement que des clichés, affirme l'impuissance de ses «prises » face à l'inexprimable, l'irréductible séparation de son art et de la douleur des victimes. Cette situation artistique extrême, à laquelle conduit le récit, et la polysémie du mot cliché, qui navigue et glisse constamment dans le texte entre deux acceptions: «lieu commun" et «instantané photographique», font de l'ouvrage une réflexion sur la séparation du sujet et du monde et sur la valeur et la possibilité de toute représentation.

Si des stéréotypes contaminent plus d'un passage, la photographie imprègne, elle, toute la narration. Les notations concernant la luminosité et l'éclairage abondent; plus d'une séquence est rapportée à un plan: «Zoom sur la nuque de Keiko (utiliser un objectif tamron 35/80)» (ÉR, 43); «Zoom sur main baladeuse se fermant sur une paire de boucles d'oreille»; «Plan intérieur» (ÉR, 152). Si bien que les scènes font souvent l'effet de mises en scène. Quant aux protagonistes de l'histoire, ils ont tous, peu ou prou, un lien avec la photographie. Hoki est photographe de mode, le narrateur ne sort qu'un «Contax 139» à la main $(\bar{E} R, 58)$ pour prendre des clichés de femmes (ER, 58-62), et de lolitas, dont il aimerait bien, en outre, développer les poses dans sa «chambre noire» (ERR, 99). Enfin, notons que tous les Japonais évoqués dans le texte sont des photographes (ou des photographiês). «Clin d'œil» malicieux à l'image stéréotypée par excellence d'un touriste nippon "photophage ${ }^{44}$ » qui "port[e] toujours deux paires de caméras au $\operatorname{cou}^{45}$ » ? Sans nul doute. Mais ce phénomène généralisé de prises de vue, qui développe dans le texte un voyeurisme, à défaut de voyage, transforme également l'Amérique du Nord en "une montagne de clichés ${ }^{46}$ »: dames d'Outremont stéréotypées (ÉR, 59), Rockefeller Center devenu à jamais une image figée sous les flashes des touristes $(\bar{E} R, 166)$, etc.

$$
++
$$

44 Georges Perec, Tentative d'épuisement d'un lieu parisien, Paris, Christian Bourgois, 1975, p. 41.45 Dany Laferrière, Cette grenade dans la main du jeune Nègre est-elle une arme ou un fruit?, op. cit., p. 76.46 Ibid., p. 19. 
Est-ce à dire que l'on ne peut jamais sortir du cliché? Qu'entre la préreprésentation du stéréotype et la reproduction photographique, on est réduit à l'image à défaut de la chose? Le texte semble trouver une résolution à cette aporie toute philosophique.

À défaut de signifier l'autre et de voir l'ailleurs par un discours rationnel et une vision claire, un autre mode d'appréhension est adopté: le haïku ${ }^{47}$, genre poétique japonais traditionnel. Texte très bref, fait d'une juxtaposition elliptique de trois vers (sans rimes, ni assonances), il est un instantané poétique, une sorte d'équivalent verbal du polaroïd. Le haïku est d'ailleurs caractéristique du récit de voyage japonais où il a longtemps figuré « un peu comme une pause au tournant du chemin ${ }^{48}$ ». Mais il est un polaroïd particulier: «le flash du haïku n'éclaire, ne révèle rien; il est celui d'une photographie que l'on prendrait très soigneusement (à la japonaise), mais en ayant omis de charger l'appareil de sa pellicule» $(E S, 111)$.

Parmi un fatras de références occidentales et de symboles tapageurs issus de la culture américaine (John Lennon, Art Garfunkel, Leonard Cohen, etc.) s'inscrit, comme à contre courant, la présence d'une littérature asiatique ténue, paisible et pacifique. Si le philosophe Lao-tseu et son Tao tö king ${ }^{49}$ imprègnent le texte d'une sagesse zen venue de Chine, c'est la poésie qui prime, avec force renvois aux maîtres du haïku. La troisième épigraphe donne le ton, avec un poème de Sora, disciple de Matsuo Bashô (1644-1694), considéré comme le maître du genre. Ces poèmes concis se retrouvent au début et à la fin de l'ouvrage. Amorcés par une référence à Bashô:

Pour faire l'amour avec Hoki il faut connaître Basho [...]. Hoki me lit au petit matin ce poème de Basho:

Éclat de la lune

J'ai passé ma nuit à tourner

Autour de l'étang $(\bar{E} R, 17)$

ils se poursuivent par des citations de Kobayashi Issa ( $E R, 26)$, de Jôgo (ÉR, 58) et de Moichi $(\bar{E} R, 61)$. Mais ils se concentrent surtout dans la dernière partie, comme un bouquet final: Buson, Kagami Shikô (ÉR, 164), Issa, Chiyo-ni (ÉR, 166) et Tôfu $(E R, 168)$. Douze poésies minimalistes datant des dix-septième et dix-huitième siècles sont ainsi reproduites, substituant à l'explication logique, une illustration fugitive du moment présent. Le narrateur lui-même s'essaie à de semblables compositions : "Le téléphone calé au pied du lit, posé sur un volume de Mishima, il pleut $^{50}$.» $(\bar{E} R, 20)$

Le choix du haiku est intéressant à plusieurs titres. Souvent saisonnier, il exprime presque toujours un lien avec la nature. «Pluie fine de printemps/une fille apprend/au chat la danse (Issa)». "Sur le ruisseau/elle court après son reflet/la

$$
+++
$$

47 C'est à Musaoka Shiki (1867-1902), père du haïku moderne, que l'on doit l'appellation «haïku ». 48 Voir Maurice Coyaud, Fourmis sans ombre, le livre du haiku, Paris, Phébus, 1978, p. 13. 49 Lao-tseu, Tao tö King, traduction de Liou Kia-Hway, Paris, Éditions Gallimard, 1967. 50 Rappelons que le premier pseudonyme de Bashô («bananier» en japonais) fut Tosei, surnom identique à celui que donne au narrateur son amante. 
libellule (Chiyo-ni)». Il constitue donc un pont entre le dedans et le dehors, l'intérieur et l'extérieur. Il traverse, en quelque sorte, la fenêtre, fidèle en cela à la philosophie zen qu'il véhicule et qui tente d'abolir la séparation entre sujet et objet, pour trouver une unité et une harmonie primordiales entre l'être et le monde.

En outre, le haïku, contrairement à la poésie occidentale, n'est pas métaphorique, il se lit à la lettre, sans intermédiaire symbolique. Enfin il n'a rien à voir avec la rationalité discursive, puisque ce n'est pas dans la déduction logique, mais dans la juxtaposition de ses trois vers que doit se produire une illumination du sens. C'est le "satori», qui ravissait autrefois Roland Barthes à la recherche d'un moyen d'expression différent de celui d'une littérature occidentale obligée de produire des significations.

Non seulement ces petites pièces ponctuent le récit, mais le texte entier tente de se rapprocher de cet exercice de poésie japonaise par sa composition fragmentaire et par la parataxe généralisée qui le touche. La juxtaposition de parties, de paragraphes, de bribes et de phrases remplace toute transition et enchaînement logique, préférant à l'explication le tableau descriptif ou la notation passagère. Ainsi, dans ce récit où perce la "quête d'un moi dans un monde éclaté ${ }^{51}$ ", à défaut d'une véritable fusion érotique, c'est dans un mimétisme stylistique, dans l'adoption d'un mode d'expression oriental que le narrateur «réalise» sa relation avec l'autre et avec le monde extérieur ${ }^{52}$.

\section{CONCLUSION}

La publication du premier ouvrage de Laferrière avait provoqué un choc dans les milieux littéraire et universitaire québécois. Tout en reposant sur des clichés grotesques que certains jugèrent douteux, sectaires et simplistes, Laferrière cristallisait dans Montréal, de façon abrupte et soudaine, un Occident blanc et raciste. Eroshima n'est certainement pas étranger aux réactions antérieures que l'auteur a suscitées. Laferrière y exhibe à nouveau un lot d'images toutes faites, mais le propos de l'écrivain semble se nuancer et s'approfondir.

En prenant pour motif les représentations consacrées, mâtinées de fantasmes d'un Extrême-Orient, avec lequel, en tant qu'Haïtien, il n'a pas d'histoire commune ni de passé mal digéré, Laferrière poursuit une réflexion sur le stéréotype, tout en s'écartant de représentations manichéennes préjudiciables. D'autant que le Japon est un Orient particulier. Victime de l'arme nucléaire, certes, il n'en demeure pas moins, à l'opposé de cet Orient passif et courbé sous le joug colonialiste, venu du dix-neuvième siècle, une nation conquérante, au passé impérialiste et au présent de grande puissance capitaliste.

$$
+++
$$

51 Ursula Mathis-Moser, Dany Laferrière, la dérive américaine, op. cit., p. 99.52 Éroshima apparaît comme une première étape de l'auteur sur un trajet zen. En effet, sept ans plus tard, Chronique de la dérive douce (Montréal, VLB éditeur, 1994) composé de trois cent soixante-cinq brefs paragraphes (comme autant de jours annuels) sera totalement imprégné de ce style éphémère et poétique. 
Mise en scène généralisée et ostentatoire d'éléments ethniques, culturels et érotiques éculés, le récit, loin d'être simplement cocasse ou provocateur, s'avère une herméneutique du stéréotype. En associant topoï et photos, par le biais du mot ambivalent "cliché», Laferrière lie images préconstruites et images venues d'une opération touristique ou d'un travail artistique. Il pousse ainsi à leur limite le problème de la connaissance et de la représentation de l'autre ou de l'ailleurs, en montrant qu'au-delà de ses implications politiques, idéologiques ou raciales, le cliché relève d'un problème ontologique et cognitif, car tout sens et toute image fixe, voire naturalise. 